\title{
International external quality assessment scheme for the laboratory diagnosis of diphtheria
}

\author{
KATHRYN H. ENGLER, ROMAN S. KOZLOV*, STEVEN J. COPPING ${ }^{\dagger}$, MEMBERS OF THE \\ EUROPEAN LABORATORY WORKING GROUP ON DIPHTHERIA and ANDROULLA EFSTRATIOU
}

Respiratory and Systemic Infection Laboratory and $\uparrow$ Quality Assurance Laboratory, PHLS Central Public Health Laboratory, 61 Colindale Avenue, London NW9 5HT and*Institute of Antimicrobial Chemotherapy, Smolensk State Medical Academy, Smolensk, Russia

\begin{abstract}
An international external quality assessment (EQA) scheme has been established so as to evaluate the proficiency of specialist, national diphtheria reference laboratories in the laboratory diagnosis of diphtheria. Six simulated clinical specimens were freeze-dried and distributed to 23 participants in 20 countries. Participants were asked to isolate, identify and perform toxigenicity testing on any corynebacteria present and to complete a simple questionnaire describing the procedures and reagents used. Only three laboratories obtained correct biochemical and toxigenicity results for all six specimens. The majority of laboratories performed better with toxigenicity testing than with the biochemical identification. Of concern were the results from three laboratories that failed to isolate any corynebacteria from four or more of the specimens. In one centre this was shown to be due to lack of availability of 'in-date' media and, in the other two, was presumed to be due to lack of experience in primary laboratory diagnostics for this organism. It is essential that countries, globally, maintain awareness and laboratory capabilities in this specialised area of microbiology. EQA is an invaluable process, which enables laboratories to monitor, evaluate and improve their own performance in such areas.
\end{abstract}

\section{Introduction}

Diphtheria is an acute infectious disease caused by toxigenic Corynebacterium diphtheriae and, more rarely, C. ulcerans. In many countries, where mass immunisation programmes have been successful in eradicating indigenous diphtheria, there is much debate about the need to screen throat specimens for these organisms [1]. The rarity of cases, alongside the everincreasing need for diagnostic laboratories to be cost effective, has resulted in many laboratories ceasing screening for these organisms. Consequently, laboratory expertise in the isolation, identification and toxigenicity testing of potentially toxigenic corynebacteria has diminished.

With the increase in global travel, the existence of diphtheria anywhere in the world represents a threat to non-immunised persons and those with low levels of immunity. The recent resurgence of diphtheria within the Newly Independent States (NIS) of the former

Received 23 March 2001; accepted 14 May 2001.

Corresponding author: Dr K. H. Engler (e-mail: kengler@ phls.nhs.uk).
Soviet Union [2] and other countries has highlighted the need for accessible and reliable laboratory diagnosis and has indicated the importance of increased clinical and microbiological awareness in both epidemic and non-epidemic countries. Indeed, in countries where diphtheria is uncommon, clinical diagnosis may be difficult and the laboratory plays a key role in providing simple, rapid and reliable methods to assist clinicians in making the correct diagnosis and to eliminate suspected but negative cases from further control measures. Therefore, it is important that each country maintains, or has easy access to, a laboratory with expertise in diphtheria diagnostics. In order to monitor and evaluate the proficiency of specialist national diphtheria reference laboratories, an international EQA scheme for laboratory diagnosis of diphtheria has been established, within the remit of the European Laboratory Working Group on Diphtheria (ELWGD).

\section{Materials and methods}

Six freeze-dried simulated clinical throat specimens were prepared as described previously [3]. Five speci- 
mens contained a Corynebacterium sp. and one or more throat commensals; specimen 4 contained throat commensals only. Corynebacterium spp. representing the various potentially toxigenic species and biotypes were selected; both toxigenic and non-toxigenic isolates were included. Normal bacterial flora were added to simulate a typical throat specimen. The organisms present in each specimen are given in Table 1. Quality control (QC) of the specimen was performed before freeze-drying (so as to ensure that the strains were representative of the species/biotype) and after freeze-drying (to ensure that the final freeze-dried specimens were not contaminated and that the pathogen was present in sufficient number for microbiological detection). Further QC was also performed after distribution of the specimens to ensure that they had remained viable.

The freeze-dried samples were despatched to 23 laboratories, in 20 countries. Participants were asked to isolate, identify and perform toxigenicity testing on any corynebacteria present and to complete a simple questionnaire to provide details of the procedures used in their laboratory.

\section{Results}

Reports were received from all 23 laboratories within the specified deadline $(2$ months from receipt of specimens). The intended result and a summary of the participant reports for each specimen are presented below and in Table 1 .

\section{Specimen 1. Intended result: toxigenic C. diphtheriae var. mitis}

Twenty laboratories reported acceptable results for this specimen. Fourteen laboratories obtained correct results for this specimen and six laboratories reported the correct toxigenicity result, but an incorrect biochemical identification. Four laboratories reported an incorrect biotype $-C$. diphtheriae biotype gravis (2), intermedius (1), belfanti (1) - one laboratory did not specify the biotype and one laboratory did not perform biochemical identification and reported the isolate as a toxigenic Corynebacterium sp. Three laboratories failed to detect the presence of a toxigenic isolate in this specimen; two laboratories were unable to isolate corynebacteria and one laboratory reported Rhodococcus spp. only.

\section{Specimen 2. Intended result: C. pseudodiphtheriticum}

Twelve laboratories obtained the correct result for this specimen. Four laboratories misidentified the isolate biochemically as another non-toxigenic species of Corynebacterium - C. xerosis (2), C. amycolatum (1), C. bovis (1) - and one laboratory did not perform biochemical identification and reported the isolate as a non-toxigenic Corynebacterium sp. Five laboratories were unable to isolate a Corynebacterium sp. from this specimen and one laboratory reported a Rhodococcus $\mathrm{sp}$. None of the laboratories misidentified the isolate as any of the potentially toxigenic species or reported a positive toxigenicity result.

\section{Specimen 3. Intended result: toxigenic C. ulcerans}

Twenty laboratories reported acceptable results for this specimen. Fifteen laboratories obtained fully correct results and five laboratories obtained the correct toxigenicity result, but reported an incorrect biotype result. Two laboratories identified the specimen as $C$. pseudotuberculosis, two laboratories as $C$. diphtheriae and one laboratory reported the isolate as a potentially toxigenic species but did not perform species identification. Of concern were the results from three laboratories that failed to detect the presence of a toxigenic organism in this specimen. Two laboratories were unable to isolate a Corynebacterium sp. from this specimen and one laboratory, despite identifying the isolate as C. pseudotuberculosis, a potentially toxigenic species, did not perform a toxigenicity test.

\section{Specimen 4. Intended result: negative (corynebacteria not isolated)}

Twenty-one of the 23 laboratories obtained the correct result for this specimen. Two laboratories reported other Corynebacterium spp. (C. striatum and a diphtheroid). Interestingly, both these laboratories did not isolate a corynebacterium from specimen 2 , which may suggest a possible transcription error between specimens 2 and 4 within these laboratories.

\section{Specimen 5. Intended result: toxigenic \\ C. diphtheriae var. gravis and non-toxigenic C. diphtheriae var. mitis}

Seventeen laboratories obtained an acceptable result for this specimen and detected the presence of a toxigenic organism. However, only four laboratories reported the presence of both a toxigenic and a non-toxigenic organism and one of these laboratories was unable to differentiate the biotypes. Five laboratories reported the presence of a non-toxigenic organism only (all biotype mitis) and did not detect the toxigenic organism in the specimen.

\section{Specimen 6. Intended result: non-toxigenic, tox-gene bearing $C$. diphtheriae var. mitis}

Fourteen laboratories obtained the correct result for this specimen (non-toxigenic $C$. diphtheriae var. mitis). Six of these fourteen laboratories performed PCR on the isolate, in addition to performing the Elek test and correctly identified this isolate as possessing the tox 
Table 1. Summary of results for each of the EQA specimens for 23 participating laboratories

\begin{tabular}{|c|c|c|c|c|c|}
\hline \multirow[b]{2}{*}{$\begin{array}{l}\text { Speciman } \\
\text { no. }\end{array}$} & \multirow[b]{2}{*}{$\begin{array}{l}\text { Intended result: organisms in speciamn } \\
\text { (other organisms present) }\end{array}$} & \multicolumn{3}{|c|}{ Number of laboratories with } & \multirow[b]{2}{*}{ Comment } \\
\hline & & $\begin{array}{l}\text { fully } \\
\text { correct } \\
\text { results }\end{array}$ & $\begin{array}{l}\text { acceptable } \\
\text { result }\end{array}$ & $\begin{array}{l}\text { unacceptable } \\
\text { result }\end{array}$ & \\
\hline 1 & $\begin{array}{l}\text { Toxigenic } C \text {. diphtheriae var. mitis } \\
\text { (Staphylococcus hominis, } \\
\text { Streptococcus oralis) }\end{array}$ & 14 & 20 & 3 & Three laboratories failed to identify a toxigenic corynebacterium \\
\hline 2 & $\begin{array}{l}\text { C. pseudodiphtheriticum } \\
\text { (Enterococcus faecalis, } \\
\text { Streptococcus salivarius) }\end{array}$ & 12 & 23 & 6 & $\begin{array}{l}\text { No laboratories reported the identification of a potentially toxigenic } \\
\text { species or a false positive toxigenicity result }\end{array}$ \\
\hline 3 & $\begin{array}{l}\text { Toxigenic C. ulcerans } \\
\text { (Staphylococcus epidermidis, } \\
\text { Streptococcus salivarius) }\end{array}$ & 15 & 20 & 3 & $\begin{array}{l}\text { Two laboratories identified this specimen as C. pseudotuberculosis. One } \\
\text { did not perform a toxigenicity test on this potentially toxigenic species. } \\
\text { Two laboratories failed to isolate a toxigenic organism }\end{array}$ \\
\hline 4 & $\begin{array}{l}\text { Corynebacterium spp. not isolated } \\
\text { (Staphylococcus epidermidis, } \\
\text { Streptococcus oralis) }\end{array}$ & 21 & 23 & 2 & No laboratories reported a false positive toxigenicity result \\
\hline 5 & $\begin{array}{l}\text { Toxigenic C. diphtheriae var. gravis and non-toxigenic } \\
\text { C. diphtheriae var. mitis } \\
\text { (Streptococcus salivarius) }\end{array}$ & 3 & 17 & 5 & $\begin{array}{l}\text { Four laboratories detected the presence of a toxigenic and a non-toxigenic } \\
\text { corynebacterium in this specimen, one of which was unable to } \\
\text { differentiate the biotypes }\end{array}$ \\
\hline 6 & $\begin{array}{l}\text { Non-toxigenic tox-gene bearing } \\
\text { C. diphtheriae var. mitis. } \\
\text { (Streptococcus salivarius) }\end{array}$ & 6 & 14 & 7 & $\begin{array}{l}\text { Five laboratories incorrectly reported a positive toxigenicity result. Three } \\
\text { laboratories performed only PCR and two laboratories reported false } \\
\text { positive phentoypic results. Two laboratories were unable to isolate a } \\
\text { Corynebacterium sp. }\end{array}$ \\
\hline
\end{tabular}


gene. Two further laboratories also obtained the correct phenotypic toxigenicity result, but obtained an incorrect biotype result. Five laboratories reported a false positive toxigenicity result for this specimen. In three of these laboratories, PCR was the only toxigenicity test performed (a phenotypic toxin test was not performed). Of greater concern were the results from two laboratories that performed a phenotypic test only and incorrectly identified the organism as toxigenic and also the two laboratories that were unable to isolate a Corynebacterium sp.

\section{Results from the questionnaires}

Media used. All 23 laboratories used Columbia blood agar for primary culture of the specimens and 22 of 23 laboratories also used a selective medium. The majority (21) of laboratories used a tellurite-containing medium as recommended in the recent guidelines [1,4]. Five laboratories used Hoyles' tellurite agar, prepared according to the WHO manual [4] and eight laboratories used an in-house tellurite-containing medium. Six laboratories used Corynebac agar (NPO 'Nutrient Media', Obolensk, Moscow Region, Russia), two laboratories used Clauberg II agar (in-house medium, prepared as described in Order No. 575 of the Ministry of Heath of the Russian Federation). The source of blood used in the media also varied. Ten laboratories used sheep blood, five used horse blood, one used cow blood and seven laboratories used human blood. Nineteen laboratories regarded colonial morphology as an important feature of microbiological diagnosis.

Microscopy. Twenty of 23 laboratories used microscopy as a primary screening method for suspect colonies from primary culture plates. Fourteen laboratories used only Gram's stain and four laboratories performed the methylene blue stain in conjunction with Gram's stain. Two laboratories performed a methylene blue stain only and three laboratories did not perform microscopy. None of the laboratories used Albert's stain as part of their procedures.

Screening tests. Two screening tests are recommended for the rapid differentiation of potentially toxigenic and non-toxigenic species $[1,4]$ : the detection of the enzymes cystinase and pyrazinamidase. Ten laboratories used both screening tests, four laboratories used pyrazinamidase alone, six used cystinase only and three did not use either. Of 14 laboratories that used the pyrazinamidase test, five performed the test as recommended in the WHO manual [4], three used the pyrazinamidase result from the API Coryne kit (bioMérieux, France) and one used chromogenic tablets (Rosco Diagnostics, Denmark). The remaining five laboratories did not specify the method used. For detection of cystinase activity, eight laboratories used Tinsdale agar, as specified in the WHO manual and seven laboratories used Pizu agar (in-house, prepared as described in Order No. 580 of the Ministry of Health of the Russian Federation).

Biochemical characterisation. Twenty-two of 23 laboratories performed biochemical characterisation on the isolates. One laboratory did not perform biotyping and reported the presence or absence of a 'potentially toxigenic Corynebacterium sp.'. This laboratory stated that they usually use the API Coryne and other biochemical methods for biotyping isolates, but these methods were not used for the EQA specimens, because of financial constraints. Fifteen laboratories used a commercial method (API Coryne) for biotyping strains and, of these, 12 used conventional tests in addition to the commercial system. Seven laboratories used conventional biochemical tests only and three laboratories used chromogenic tablets (Rosco) in conjunction with other tests. Conventional biochemical tests, performed by 18 laboratories, were primarily sugar fermentation and detection of urease and nitrate. Various combinations of sugars were used and these included glucose, sucrose, maltose and glycogen or starch, as recommended in the WHO manual. The majority of laboratories (14) used Hiss serum water sugars for detecting sugar fermentation.

Toxigenicity testing. Twenty-one of 23 laboratories performed tests for the detection of diphtheria toxin. The two laboratories that did not undertake toxigenicity testing were not official National Diphtheria Reference Centres for their country, and reported that they referred isolates to their national or state reference laboratory for toxigenicity testing. Nineteen laboratories performed the Elek test $[4,5]$ for detection of toxigenicity and, of these, seven also used PCR detection of the toxin gene (fragment A) $[6,7]$. Two laboratories used PCR alone and did not perform a phenotypic toxigenicity test.

Media, reagents and control strains used for the Elek test were variable. Eight laboratories prepared their Elek base according to WHO recommendations and 11 laboratories used a commercial medium for their Elek tests. Eight laboratories used Corynetox agar (NPO 'Nutrient Media', Obolensk, Moscow Region, Russia), one laboratory used Statens Serum Institut (SSI) Diagnostika Media (SSI, Denmark) and two laboratories used KL Virulence Agar (Difco).

The majority (17) of laboratories prepared strips and disks for use in the Elek test with diphtheria antitoxin at a concentration of $500 \mathrm{IU} / \mathrm{ml}$, as recommended in the WHO manual. Antitoxin was obtained from various sources including Pasteur Mérieux (Lyon, France) (eight laboratories) or NPO 'Biomed' (Perm, Russia) (six), Swiss Serum Institute (Bern, Switzerland) (two) and the Cantacuzino Institute (Bucharest, Romania) (one). The remaining two laboratories used antitoxin from Connaught Laboratories at $100 \mathrm{IU} / \mathrm{ml}$ or $\mathrm{KL}$ Antitoxin (Difco, USA) at $64 \mathrm{IU} / \mathrm{ml}$. Nine laboratories 
used antitoxin strips, three laboratories used antitoxin disks, six laboratories used both and one laboratory used antitoxin incorporated in the agar base. The majority of laboratories (15) incubated tests for a maximum of $48 \mathrm{~h}$ before reporting a final result; however, four laboratories incubated plates for up to $72 \mathrm{~h}$ before reporting a negative result. The use of control strains for the Elek test also varied. Nine laboratories used the three control strains as recommended in the WHO manual [4]. A further four laboratories used two of the three control strains (strong toxin-producer NCTC 10648 and the nontoxigenic control strain NCTC 10356 only). Two laboratories used only one control strain (strong toxin producer) and four laboratories used only 'local control strains'. Some laboratories performed additional toxigenicity tests, which included passive haemaglutination [4] (two laboratories) and an enzyme immunoassay [8] (one laboratory).

\section{Discussion}

Following the emergence of epidemic diphtheria in the NIS of the former USSR, there has been renewed interest, globally, in the laboratory diagnosis of diphtheria [9]. The international external quality assessment scheme for laboratory diagnosis of diphtheria was established so as to evaluate the proficiency of key national reference laboratories and other laboratories for the microbiological diagnosis of diphtheria. The majority of laboratories participating in the EQA scheme are specialists in the laboratory diagnosis of diphtheria and provide national reference facilities for microbiological diagnosis; therefore, the distribution was designed to challenge such laboratories.

Recommended procedures for microbiological diagnosis have been published $[1,4]$ and comprise primary isolation of suspect isolates from clinical specimens followed by biochemical identification and toxigenicity testing of the suspect isolates. The detection of toxigenicity is the most important test in the microbiological diagnosis of diphtheria, the results of which have significant impact on patient and contact management and treatment. Therefore, an incorrect toxigenicity result is not acceptable, and was considered a major error in this EQA scheme. Biotyping is of little value in patient management; therefore, such errors were considered minor. However, toxigenicity testing and biotyping can be performed only following successful primary isolation of a corynebacterium from the specimen. Therefore, failure to isolate a corynebacterium from a specimen was not acceptable and was considered a major error.

Only three laboratories reported fully correct results for all six specimens; these laboratories were all in current epidemic areas. A further nine laboratories reported ac- ceptable results for all six specimens; these laboratories isolated potentially toxigenic corynebacteria from all specimens in which they were present and obtained the correct toxigenicity result but reported minor errors for the biochemical identification. The results from three laboratories were of concern as they reported unacceptable results for three or more specimens (failure to isolate a corynebacterium or an incorrect toxigenicity result).

Specimen 5, which contained two pathogens toxigenic $C$. diphtheriae var. gravis and non-toxigenic C. diphtheriae var. mitis - was problematic for the majority (19 of 23) of laboratories. Only four laboratories correctly reported the presence of both toxigenic and non-toxigenic $C$. diphtheriae in this specimen. However, the results of an additional 14 laboratories were acceptable; these laboratories reported the presence of a toxigenic Corynebacterium sp. only in this specimen and, therefore, for diagnostic, treatment and clinical purposes, obtained the correct result. Unacceptable results were reported by five laboratories, which failed to detect the presence of a toxigenic organism in this specimen. Interestingly, 16 of the 18 laboratories that reported only a toxigenic organism reported the biotype as gravis, whereas all five laboratories that reported a non-toxigenic organism identified the biotype as mitis. This may reflect the laboratory procedure of selecting only a single suspect colony from the tellurite plate, which is subcultured and subsequently used for toxigenicity and identification tests. However, it is recommended [1,4] that several individual suspect colonies should be selected and used for subsequent tests, in particular toxigenicity testing.

Specimen 6 was also challenging, as it contained a non-toxigenic tox-gene bearing (NTTB) C. diphtheriae. Such isolates possess the diphtheria toxin gene (toxigenic in PCR-based assays) but do not express a biologically active form of the toxin (non-toxigenic by phenotypic assays) and for diagnostic purposes are considered non-toxigenic. Obtaining the correct result for this specimen was, therefore, dependent upon the methodologies used for toxigenicity testing. The use of both phenotypic and genotypic toxigenicity tests was necessary to accurately identify this NTTB isolate. The use of a phenotypic toxigenicity test alone enabled a correct toxigenicity result for diagnostic purposes, whereas the use of genotypic (PCR) methods alone resulted in a false positive toxigenicity result. Sixteen laboratories obtained acceptable results for this specimen and reported the presence of a non-toxigenic isolate. Six of these laboratories also performed PCR and reported the presence of a NTTB isolate. Four laboratories reported unacceptable results for this specimen and reported a false positive toxigenicity result. For two laboratories this was because they used PCR alone for toxigenicity testing and did not perform a phenotypic toxin test. Although NTTB strains may be 
relatively uncommon, they are being isolated with increasing frequency in countries of the former USSR, particularly in areas where the epidemic is declining [6]. Current recommendations are that PCR should be used only in conjunction with a phenotypic toxigenicity test $[1,4,9]$. The two remaining laboratories used only a phenotypic toxigenicity test (Elek test) and obtained a false positive result, which was of major concern. The reasons for this are unclear. One laboratory reported that the Elek plates were incubated for $>48 \mathrm{~h}$; this could produce non-specific precipitin lines and, therefore, a false positive result [10]. However, this is not consistent with this laboratory's performance with the other specimens (correct results for specimens 1-4 and detected the toxigenic strain in specimen 5).

The results from three laboratories were of major concern. These laboratories were unable to isolate any corynebacteria from three (two laboratories) or four (one laboratory) of the five specimens that contained corynebacteria. In one laboratory this was proved to be due to the use of 'out of date' media. This laboratory reported that because of problems with media availability, it had been necessary to prepare primary isolation plates from dehydrated media produced in the 1980s. For two other laboratories, the reason for their poor performance is unclear. Both laboratories reported using methods as recommended in the WHO manual, and one of the laboratories used media and reagents provided by the WHO Collaborating Centre in London. Therefore, it seemed unlikely that their problems were due to media and reagents. However, both laboratories are in 'non-epidemic areas' and have only recently become members of the ELWGD. These laboratories lack extensive experience in laboratory diagnosis of diphtheria and, therefore, EQA schemes such as these are extremely beneficial. They enable the laboratories not only to monitor their performance, but also to increase their experience in dealing with organisms that are not routinely isolated in their country.

Of concern was another laboratory that reported the presence of $C$. pseudotuberculosis in specimen three (which contained $C$. ulcerans), but did not perform a toxigenicity test on this isolate. C. ulcerans and $C$. pseudotuberculosis are biochemically similar, but can be differentiated easily on the basis of differences in glycogen fermentation, trehalose hydrolysis and gelatin liquefaction. More importantly, both species are potentially toxigenic. Toxigenicity testing should always be performed on such isolates.

Generally, the majority of laboratories performed better with toxigenicity testing than biochemical identification. Of 138 reports for toxigenicity and biochemical identification (six specimens tested by 23 laboratories), $121(88 \%)$ toxigenicity test reports were correct compared with $85(62 \%)$ biochemical identification reports.
Of concern was the lack of use of appropriate control strains, in particular for the cystinase and Elek test. Six of the 17 laboratories that performed the cystinase test, used local control strains (often toxigenic), rather than the non-toxigenic NCTC/ATCC control strain recommended in guidelines [4]. Similarly, for the Elek test, five of 19 laboratories performing the Elek test used local strains rather than recommended control strains [4].

The methodologies used by the participants for microbiological diagnosis of diphtheria were mainly as recommended in the WHO guidelines [4]. Standardisation of methodologies probably reflects the close working relationships and collaborations that exist between the participants. The majority of participants are members or collaborators of the ELWGD and many are partners within the EU funded programmes for Microbiological Surveillance of Diphtheria in Europe. These programmes have enabled the organisation of workshops and training visits for diphtheria diagnostics and thus have contributed significantly to the standardisation of methodologies amongst members [11].

Overall the results of the EQA distribution were encouraging. However, there is clearly a continued need for, at least, yearly distributions of specimens for external quality assessment within laboratories worldwide. This will enable laboratories to monitor performance and maintain awareness and capabilities within this increasingly important and specialised area of microbiology.

We thank all colleagues for their participation and Dr R. C. George for critical review of the manuscript. This work was funded in part by the European Commission DG RTD programmes: BioMed 2 BMH4.CT. 98.3793 'Microbiological surveillance of diphtheria in Europe' and INCO Copernicus IC15.CT98.0302 'Microbiological surveillance of diphtheria in Eastern Europe'.

\section{References}

1. Efstratiou A, George RC. Laboratory guidelines for the diagnosis of infections caused by Corynebacterium diphtheriae and C. ulcerans. Comm Dis Pub Hlth 1999; 2: 250-257.

2. Galazka A. The changing epidemiology of diphtheria in the vaccine era. J Infect Dis 2000; 181 Suppl 1: S2-S9.

3. Snell JJS, De Mello JV, Gardner PS. The United Kingdom national microbiological quality assessment scheme. $J$ Clin Pathol 1982; 35: 82-93.

4. Efstratiou A, Maple PAC. Manual for the laboratory diagnosis of diphtheria. The Expanded Programme on Immunisation in the European Region of WHO 1994. ICP/EPI 038(C).

5. Engler KH, Glushkevich TG, Mazurova IK, George RC, Efstratiou A. A modified Elek test for the detection of toxigenic corynebacteria in the diagnostic laboratory. $J$ Clin Microbiol 1997; 35: 495-498.

6. Pallen MJ. Rapid screening for toxigenic Corynebacterium diphtheriae by the polymerase chain reaction. J Clin Pathol 1991; 44: 1025-1026.

7. Mikhailovich VM, Melnikov VG, Mazurova IK et al. Application of PCR for detection of toxigenic Corynebacterium diphtheriae strains isolated during the Russian diphtheria epidemic, 1990 through 1994. J Clin Microbiol 1995; 33: 3061-3063.

8. Engler KH, Efstratiou A. Rapid enzyme immunoassay for 
determination of toxigenicity among clinical isolates of corynebacteria. J Clin Microbiol 2000; 38: 1385-1389.

9. Efstratiou A, Engler KH, Mazurova IK, Glushkevich TG, Vuopio-Varkila J, Popovic T. Current approaches to the laboratory diagnosis of diphtheria. J Infect Dis 2000; 181 Suppl 1: S138-S145.
10. Colman G, Weaver E, Efstratiou A. Screening tests for pathogenic corynebacteria. J Clin Pathol 1992; 45: 46-48.

11. Efstratiou A, Roure $\mathrm{C}$ and Members of the European Laboratory Working Group on Diphtheria. The European Laboratory Working Group on Diphtheria: a global microbiologic network. J Infect Dis 2000; 181 Suppl 1: S146-S151. 